\title{
The Stationary Distribution of Competitive Lotka-Volterra Population Systems with Jumps
}

\author{
Zhenzhong Zhang, ${ }^{1}$ Jinying Tong, ${ }^{1}$ and Jianhai Bao ${ }^{2}$ \\ ${ }^{1}$ College of Science, Donghua University, Shanghai 201620, China \\ ${ }^{2}$ Department of Mathematics, Central South University, Changsha 410075, China \\ Correspondence should be addressed to Zhenzhong Zhang; zzzhang@dhu.edu.cn \\ Received 17 December 2013; Accepted 16 January 2014; Published 13 March 2014 \\ Academic Editor: Litan Yan
}

Copyright (c) 2014 Zhenzhong Zhang et al. This is an open access article distributed under the Creative Commons Attribution License, which permits unrestricted use, distribution, and reproduction in any medium, provided the original work is properly cited.

Dynamics of Lotka-Volterra population with jumps (LVWJ) have recently been established (see Bao et al., 2011, and Bao and Yuan, 2012). They provided some useful criteria on the existence of stationary distribution and some asymptotic properties for LVWJ. However, the uniqueness of stationary distribution for $n \geq 2$ and asymptotic pathwise estimation $\lim _{t \rightarrow+\infty}(1 / t) \int_{0}^{t}|X(s)|^{p} d s(p>0)$ are still unknown for LVWJ. One of our aims in this paper is to show the uniqueness of stationary distribution and asymptotic pathwise estimation for LVWJ. Moreover, some characterizations for stationary distribution are provided.

\section{Introduction}

Recently, Bao et al. [1] introduced a jump process into the underlying population dynamics to characterize sudden environmental shocks, for example, earthquakes, hurricanes, epidemics, and so forth. They assume that population sizes follow the following stochastic differential equations:

$$
\begin{aligned}
d X(t)= & \operatorname{diag}\left(X_{1}(t), \ldots, X_{n}(t)\right) \\
\times & {\left[\left(a(t)-B(t) X\left(t^{-}\right)\right) d t+\sigma(t) d W(t)\right.} \\
& \left.+\int_{\mathbb{Y}} \gamma(t, u) \widetilde{N}(d t, d u)\right],
\end{aligned}
$$

where $X\left(t^{-}\right)$stands for the left limit of $X(t), X(t)=$ $\left(X_{1}(t), \ldots, X_{n}(t)\right)^{T}, B(t)=\left(b_{i j}(t)\right)_{n \times n}, \sigma(t)=\left(\sigma_{i j}(t)\right)_{n \times n}$, and $\gamma(t, u)=\left(\gamma_{1}(t, u), \ldots, \gamma_{n}(t, u)\right)$. Here $b_{i j}(t)$ represents the effect of interspecies (if $i \neq j$ ) or intraspecies (if $i=$ $j)$ interaction and $W(t)=\left(W_{1}(t), \ldots, W_{n}(t)\right)^{T}$ is an $n$ dimensional Brownian motion. $N(d t, d u)$ is a Poisson counting measure with characteristic measure $\lambda$ on a measurable subset $\mathbb{Y}$ of $[0,+\infty)$ with $\lambda(\mathbb{Y})<\infty$ and $\widetilde{N}(d t, d u):=$ $N(d t, d u)-\lambda(d u) d t$. Besides, we suppose that $W(t)$ and $N(t)$ are independent and for $i, j=1, \ldots, n, \sigma_{i j}(t)$ are nonnegative constants. If $b_{i i}(t)>0, b_{i j}(t) \leq 0,1 \leq i, j \leq n, i \neq j$, then the model (1) is termed as the facultative Lotka-Volterra model with jumps. If $b_{i i}(t)>0, b_{i j}(t)>0,1 \leq i, j \leq n, i \neq j$, then the model (1) is termed as the competitive Lotka-Volterra model with jumps. Bao et al. [1,2] reveal some important and nice properties.

(i) Jump processes can suppress the explosion.

(ii) Under Assumption A1 (see Section 2), there exists an invariant measure for the solution of population model (1).

(iii) When the white noise and sudden noise are large, stochastic population dynamics (1) tend to be extinct. To be precisely, under Assumptions A1 and A2 (see Section 2), the population dynamics (1) tend to be extinct.

(iv) When the white noise and sudden noise are small, stochastic population dynamics (1) is stochastically permanent and has a unique stationary distribution.

It is a natural question that when the white noise and sudden noise are small do the population dynamics (1) have a unique stationary distribution for $n \geq 2$ ? If yes, can we give 
some characterizations of the stationary distribution? In this paper, we shall show that the population dynamics have the following properties.

(i) Under Assumption A1 (see Section 2), there exists a unique stationary distribution and it has ergodic property.

(ii) Under Assumptions A1 and A3 (see Section 2), the average in time of any $p$ of the path $|X(t)|$ is bound with probability one. Besides, we have

$$
\operatorname{Lim}_{t \rightarrow \infty} \frac{1}{t} \int_{0}^{t}|X(s)|^{p} d s=\int_{\mathbb{R}_{+}^{n}}|x|^{p} \pi(d x) \leq K \text { a.s., }
$$

where $\pi$ is the stationary distribution and $p$ is a positive constant.

(iii) Some characterizations of the stationary distribution are provided.

\section{Notation}

Throughout this paper, we let $\left(\Omega, \mathscr{F},\left\{\left\{\mathscr{F}_{t}\right\}_{t \geq 0}\right\}, P\right)$ be a complete probability space with a filtration $\left\{\mathscr{F}_{t}\right\}_{t \geq 0}$ satisfying the usual conditions (i.e., it is right continuous and $\mathscr{F}_{0}$ contains all $P$-null sets). Let $|\cdot|$ denote the Euclidean norm in $\mathbb{R}^{n}$. If $A$ is a vector or matrix, its transpose is denoted by $A^{T}$. If $A$ is a matrix, its trace norm is denoted by $\sqrt{\operatorname{trace}\left(A^{T} A\right)}$. Let $\mathbb{R}^{n}$ be $n$-dimensional real Euclidean space and $\mathbb{R}_{+}^{n}$ be the set $\left\{x \in \mathbb{R}^{n}: x_{i}>0,1 \leq i \leq n\right\} ;|A|:$ the trace norm of matrix $A$; that is, $|A|:=\sqrt{\operatorname{trace}\left(A^{T} A\right)}$.

We shall need a few more notations. Define $B(y, \varepsilon)=\{x \in$ $\left.\mathbb{R}_{+}^{n}:|x-y| \leq \varepsilon\right\}, B^{c}(y, \varepsilon)=\left\{x \in \mathbb{R}_{+}^{n}:|x-y|>\varepsilon\right\}$.

Denote transition probabilities of (1) by

$$
P^{t}(x, d y)=P(X(t) \in d y \mid X(0)=x) .
$$

The transition probabilities can be thought of as operators on bounded Borel measurable functions $f: \mathbb{R}_{+}^{n} \rightarrow \mathbb{R}$ via

$$
P^{t} f(x)=\int_{\mathbb{R}_{+}^{n}} P^{t}(x, d y) f(y)
$$

Following Bao et al. [2], the following assumptions are imposed for the model (1).

Assumption A1. For any $t \geq 0$ and $i, j=1,2, \ldots, n$ with $i \neq j$, $a_{i}(t)>0, b_{i i}(t)>0, b_{i j}(t) \geq 0, \sigma_{i j}(t)$, and $\gamma_{i}(t, u)$ are bounded functions; $\sigma(t) \neq(0)_{n \times n}, \widehat{b}:=\inf _{t \in \mathbb{R}_{+}} b_{i i}(t)>0$, and $\gamma_{i}(t, u)>$ $-1, u \in \mathbb{Y}$.

Assumption A2. For $t \geq 0$ and $i=1,2, \ldots, n$, the coefficients of $X(t)$ determined by (1) obey the following condition:

$$
\begin{aligned}
\eta_{i}=\limsup _{t \rightarrow+\infty} \frac{1}{t} \int_{0}^{t}\left(a_{i}(s)-\frac{1}{2} \sum_{j=1}^{n} \sigma_{i j}^{2}(s)\right. \\
\quad-\int_{\mathbb{Y}}\left(\gamma_{i}(s, u)\right. \\
\left.\left.\quad-\ln \left(1+\gamma_{i}(s, u)\right)\right) \lambda(d u)\right) d s<0 .
\end{aligned}
$$

Assumption A3. There exists a constant $\bar{K}(p)>0$ such that for some $p>1, t \geq 0, i=1, \ldots, n$, we have

$$
\int_{\mathbb{V}}\left|\gamma_{i}(t, u)\right|^{p} \lambda(d u) \leq \bar{K}(p) .
$$

We know from Bao et al. [1] that Assumption A1 is a basic condition which guarantees that for any initial condition $X(0)=x_{0}$, (1) has a unique global positive solution; namely, $P\left(X(t) \in \mathbb{R}_{+}^{n}\right.$, for all $\left.t \geq 0\right)=1$. Besides, throughout this paper, we let $K$ be a generic positive constant whose values may vary at its different appearances.

\section{Main Results}

With the notations introduced in the previous section, we can state one of our main results.

Theorem 1. Under Assumption A1, the SDE model (1) has a unique stationary distribution and it has ergodic property.

The proof of Theorem 1 relies on the following several lemmas. The first lemma is taken from Theorem 3.1 in Bao et al. [1].

Lemma 2 (Bao et al. [1]). (1) Under Assumption A1, for any $0 \leq p \leq 1$, there is a constant $K$ such that $\sup _{t \in \mathbb{R}^{+}} E|X(t)|^{p} \leq$ K.

(2) Under Assumptions A1 and A3, for any $p>1$, there is a constant $K(p)$ such that $\sup _{t \in \mathbb{R}^{+}} E|X(t)|^{p} \leq K(p)$.

Define $\tau_{D}$ to be the first time the diffusion exists in domain $D$ :

$$
\tau_{D}=\inf \{t \geq 0 ; X(t) \notin D\} .
$$

Similar to Proposition 7.2 in Karatzas and Shreve [3, pages 364-365], we have the following lemma.

Lemma 3. Suppose that for the open, bounded domain $D$ and for some $1 \leq i \leq n$, if the following condition: $\min _{(t, x) \in \mathbb{R}^{+} \times \bar{D}} \sum_{j=1}^{n} \sigma_{i j}^{2}(t) x_{i}^{2}>0$ holds, then $E^{x} \tau_{D}<\infty$, for any $x \in D$. 
Proof. For simplicity, let $a_{i i}(t, x)=(1 / 2) \sum_{j=1}^{n} \sigma_{i j}^{2}(t) x_{j}^{2}$, $b_{i}(t, x)=a_{i}(t)-\sum_{j=1}^{n} b_{i j}(t) x_{j}$, and let $a=\min _{(t, x) \in \mathbb{R}^{+} \times \bar{D}}$ $a_{i i}(t, x), b=\max _{(t, x) \in \mathbb{R}^{+} \times \bar{D}}\left|b_{i}(t, x)\right|, q=\min _{x \in \bar{D}} x_{i}$.

Consider the function $h(x)=-\mu \exp \left(v x_{i}\right) ; x=\left(x_{1}, \ldots\right.$, $\left.x_{n}\right) \in D$, where the positive constants $v, \mu$ shall be determined later. Then, we have

$$
\begin{array}{r}
\operatorname{Lh}(x)=-\mu e^{v x_{i}}\left\{\frac{1}{2} v^{2} a_{i i}(t, x)+v b_{i}(t, x)\right. \\
+\int_{\mathbb{V}}\left(\exp \left(v x_{i} \gamma_{i}(t, u)\right)-1\right. \\
\left.\left.\quad-v x_{i} \gamma_{i}(t, u)\right) \lambda(d u)\right\} .
\end{array}
$$

Note that the inequality $e^{x}-1-x \geq 0$ for all $x \in \mathbb{R}$; then,

$$
\operatorname{Lh}(x) \leq-\frac{1}{2} \mu v a e^{v q}\left(v-\frac{2 b}{a}\right) .
$$

Consequently, if Assumption (i) holds, then we can choose $v>2 b / a$ and a sufficiently large $\mu$ such that for any $x \in D$, we have

$$
\operatorname{Lh}(x) \leq-1 \text {. }
$$

With the boundedness of the function $h$ and its derivatives on $\bar{D}$, Dnykin's formula implies, $x \in D, t \geq 0$, the following:

$$
\begin{aligned}
E^{x}\left(t \wedge \tau_{D}\right) & =h(x)+E^{x} L h(X(s)) \\
& \leq h(x)-E^{x} h\left(X_{t \wedge \tau_{D}}\right) \\
& \leq 2 \max _{y \in \bar{D}}|h(y)|<\infty .
\end{aligned}
$$

By letting $t \rightarrow \infty$, we obtain

$$
E^{x} \tau_{D}<\infty, \quad x \in D .
$$

Lemma 4. Under Assumption A1, the SDE model (1) has the (weak) Feller property.

Proof. To prove the weak Feller property, by Theorem 5.1 (see Bhattacharya and Waymire [4, pages 643-645]), we only need to show that for every bounded Lipschitzian function $f$ on $\mathbb{R}_{+}^{n}$, if $x_{0} \rightarrow y_{0}$ is well defined, we have

$$
\left|E f\left(X^{x_{0}}(t)\right)-E f\left(X^{y_{0}}(t)\right)\right| \longrightarrow 0 \text { as } x_{0} \longrightarrow y_{0} .
$$

The proof of the limit above is essentially the same as that of Lemma 3.2 in Tong et al. [5]. Consequently, we only need to prove that $x_{0} \rightarrow y_{0}$ is well defined. For any $X(0)=$ $x_{0}:=\left(x_{01}, \ldots, x_{0 n}\right)^{T}$, let $\varepsilon_{0}=(1 / 2) \min \left\{x_{01}, \ldots, x_{0 n}\right\}$. By Assumption A1, we have

$$
\min _{x \in B\left(x_{0}, \varepsilon_{0}\right)} \frac{1}{2} \sum_{j=1}^{n} \sigma_{i j}^{2}(t) x_{j}^{2}>0 .
$$

By Lemma 3, we have $E^{x_{0}} \tau_{B\left(x_{0}, \varepsilon_{0}\right)}<+\infty$, which implies that $x_{0} \rightarrow y_{0}$ is well defined.

We are now able to prove Theorem 1 .
Proof of Theorem 1. The proof of this theorem is divided into two steps.

Step 1. (Existence). Bao et al. [1] show that (1) exists as an invariant measure:

$$
P^{t}\left(x_{0}, d z\right) \longrightarrow \pi(d z), \quad \forall x_{0} \in \mathbb{R}_{+}^{n} .
$$

Step 2. (Uniqueness). By Lemma 4 and the same discussion as that of Theorem 3.1 in Tong et al. [5], one can easily prove that $\pi$ is the unique station distribution.

Lemma 5. Suppose that condition (A1) holds. If $f(x)$ is a function integrable with respect to the measure $\pi$, then

$$
P\left\{\lim _{t \rightarrow \infty} \frac{1}{t} \int_{0}^{t} f(X(s)) d s=\int_{\mathbb{R}_{+}^{n}} f(x) \pi(d x)\right\}=1 .
$$

Proof. The proof is essentially the same as the proof of Theorem 5.1 of Has'minskiu [6]. We omit its proof.

With the lemma above, we can get the following long time behavior of population system (1).

Theorem 6. If Assumptions A1 and A3 hold, for any $p>0$, then we have

$$
\begin{aligned}
& \lim _{t \rightarrow+\infty} \frac{1}{t} \int_{0}^{t} X_{i}^{p}(s) d s=\int_{\mathbb{R}_{+}^{n}} y_{i}^{p} \pi(d y), \quad \text { a.s. } \\
& \lim _{t \rightarrow+\infty} \frac{1}{t} \int_{0}^{t}|X(s)|^{p} d s=\int_{\mathbb{R}_{+}^{n}}|y|^{p} \pi(d y), \quad \text { a.s. }
\end{aligned}
$$

Proof. Let $f(x)=x_{i}^{p} \wedge k$ and $g(x)=|x|^{p} \wedge k$ for some $k>0$, where $a \wedge b=\min \{a, b\}$. Obviously, $f(x)$ and $g(x)$ are integrable with stationary distribution $\pi$. Hence, by Lemma 5 , we get

$$
\begin{aligned}
& \lim _{t \rightarrow+\infty} \frac{1}{t} \int_{0}^{t}\left(X_{i}^{p}(s) \wedge k\right) d s=\int_{\mathbb{R}_{+}^{n}}\left(y_{i}^{p} \wedge k\right) \pi(d y), \\
& \lim _{t \rightarrow+\infty} \frac{1}{t} \int_{0}^{t}\left(|X(s)|^{p} \wedge k\right) d s=\int_{\mathbb{R}_{+}^{n}}\left(|y|^{p} \wedge k\right) \pi(d y), \quad \text { a.s. }
\end{aligned}
$$

According to Lemma 2, it follows that

$$
\begin{aligned}
& E\left[\lim _{t \rightarrow+\infty} \frac{1}{t} \int_{0}^{t}\left(X_{i}^{p}(s) \wedge k\right) d s\right] \\
& =\lim _{t \rightarrow+\infty} \frac{1}{t} \int_{0}^{t} E\left(X_{i}^{p}(s) \wedge k\right) d s \leq K, \quad \text { a.s., } \\
& E\left[\lim _{t \rightarrow+\infty} \frac{1}{t} \int_{0}^{t}\left(|X(s)|^{p} \wedge k\right) d s\right] \\
& \quad=\lim _{t \rightarrow+\infty} \frac{1}{t} \int_{0}^{t} E\left(|X(s)|^{p} \wedge k\right) d s \leq K \quad \text { a.s. }
\end{aligned}
$$


From (18) and (20), (19), and (21), we get

$$
\begin{aligned}
& \int_{\mathbb{R}_{+}^{n}}\left(y_{i}^{p} \wedge k\right) \pi(d y) \leq K, \\
& \int_{\mathbb{R}_{+}^{n}}\left(|y|^{p} \wedge k\right) \pi(d y) \leq K .
\end{aligned}
$$

By letting $k \rightarrow+\infty$, it follows that

$$
\begin{gathered}
\int_{\mathbb{R}_{+}^{n}}\left(y_{i}^{p}\right) \pi(d y) \leq K, \\
\int_{\mathbb{R}_{+}^{n}}\left(|y|^{p}\right) \pi(d y) \leq K .
\end{gathered}
$$

Namely, $f_{i}(x)=x_{i}^{p}$ and $g_{i}=|x|^{p}$ are integrable with respect to the unique invariant measure $\pi(\cdot)$. Again by Lemma 5 , the proof is complete.

\section{Characterizations of Stationary Distribution}

In what follows, we consider some characterizations of the stationary distribution of (1). By Theorem 4.6 of Bao et al. [1], for $1 \leq i, j \leq n$, if

$$
\begin{aligned}
\limsup _{t \rightarrow+\infty} \frac{1}{t} \int_{0}^{t} & \left(a_{i}(s)-\frac{1}{2} \sum_{j=1}^{n} \sigma_{i j}^{2}(s)\right. \\
& \left.-\int_{\mathbb{V}}\left(\gamma_{i}(s, u)-\ln \left(1+\gamma_{i}(s, u)\right)\right) \lambda(d u)\right) d s
\end{aligned}
$$

$<0$,

then stochastic population dynamics (1) are exponential extinctions; that is the unique stationary distribution $\pi(0)=$ 1. We shall show that condition (24) also is necessary condition for exponential extinction if we replace the bounded coefficients with uniformly bounded coefficients under Assumption A1. Hence, we impose a stronger assumption on coefficients of population dynamics (1).

Assumption A4. For any $t \geq 0$ and $i, j=1,2, \ldots, n$ with $i \neq j$, $a_{i}(t)>0, b_{i i}(t)>0, b_{i j}(t) \geq 0, \sigma_{i}(t) \neq 0$, and $\gamma_{i}(t, u)$ are uniformly bounded functions on time $t, \widehat{b}:=\inf _{t \in \mathbb{R}+} b_{i i}(t)>$ 0 , and $\gamma_{i}(t, u)>-1, u \in \mathbb{Y}$.

Theorem 7. Under Assumption A4, stochastic population dynamics (1) are exponential extinctions if and only if condition (24) holds.
Proof. Sufficiency: By Itô's lemma, we have

$$
\begin{aligned}
\ln X_{i}(t)= & \ln X_{i}(0) \\
& +\int_{0}^{t}\left(a_{i}(s)-\frac{1}{2} \sum_{j=1}^{n} \sigma_{i j}^{2}(s)\right. \\
& \left.-\int_{\mathbb{Y}} \gamma_{i}(s, u) \lambda(d u)\right) d t \\
& -\sum_{j=1}^{n} \int_{0}^{t} b_{i j}(s) X_{j}(s) d s \\
& +M_{1}(t)+M_{2}(t) \\
& +\int_{0}^{t} \int_{\mathbb{Y}} \ln \left(1+\gamma_{i}(s, u)\right) \lambda(d u) d s
\end{aligned}
$$

where $M_{1}(t)=\sum_{j=1}^{n} \int_{0}^{t} \sigma_{i j}(s) d W_{j}(s), M_{2}(t)=\int_{0}^{t} \int_{\mathbb{Y}} \ln (1+$ $\left.\gamma_{i}(s, u)\right) \widetilde{N}(d s, d u)$. Under Assumption A4, the sharp bracket process is as follows:

$$
\begin{aligned}
\left\langle M_{1}\right\rangle(t)= & \sum_{i=1}^{n} \int_{0}^{t} \sigma_{i j}^{2}(s) d s \leq K t, \\
\left\langle M_{2}\right\rangle(t) & =\int_{0}^{t} \int_{\mathbb{Y}}\left(\ln \left(1+\gamma_{i}(s, u)\right)\right)^{2} \lambda(d u) d s \\
& \leq K t .
\end{aligned}
$$

By the strong law of large numbers of martingales, we have

$$
\begin{aligned}
& \lim _{t \rightarrow \infty} \frac{M_{1}(t)}{t}=0, \quad \text { a.s., } \\
& \lim _{t \rightarrow \infty} \frac{M_{2}(t)}{t}=0 \quad \text { a.s. }
\end{aligned}
$$

If stochastic population dynamics are exponential extinctions a.s., then $\lim \sup _{t \rightarrow+\infty}\left(\left(\ln X_{t}\right) / t\right)<0$, which implies the assertion.

Necessity: the proof can be found in Theorem 4.6 of Bao et al. [1].

In the rest of this paper, we consider the nontrivial stationary distribution case.

Assumption A5. We have

$$
\begin{aligned}
\limsup _{t \rightarrow+\infty} \frac{1}{t} \int_{0}^{t}\left(a_{i}(s)-\frac{1}{2} \sum_{j=1}^{n} \sigma_{i j}^{2}(s)\right. \\
\left.\quad-\int_{\mathbb{Y}}\left(\gamma_{i}(s, u)-\ln \left(1+\gamma_{i}(s, u)\right)\right) \lambda(d u)\right) d s
\end{aligned}
$$

$\geq 0$. 
Next, we consider the mean vector and covariance matrix of the stationary distribution. If we assume that Assumptions A1 and A3 hold, then Theorem 6 implies that both mean vector $\bar{\mu}=\left(\bar{\mu}_{1}, \ldots, \bar{\mu}_{n}\right)^{T}$ and covariance matrix $\Sigma=\left(\Sigma_{i j}\right)_{n \times n}$ of the stationary distribution; namely,

$$
\mu=\int_{\mathbb{R}_{+}^{n}} y \pi(d y), \quad \Sigma=\int_{\mathbb{R}_{+}^{n}}(y-\bar{\mu})(y-\bar{\mu}) \pi(d y)
$$

are well defined.

The next theorem gives an explicit formula for the mean vector of the stationary distribution of (1).

Theorem 8. If conditions $A 1$ and $A 3$ hold, then the mean vector $\bar{\mu}$ of the stationary distribution follows

$$
\begin{aligned}
\int_{0}^{t}\left(a_{i}(s)-\frac{1}{2} \sum_{j=1}^{n} \sigma_{i j}^{2}(s)-\sum_{j=1}^{n} b_{i j} \mu_{j}(s)\right) d s \\
\quad-\int_{0}^{t}\left(\int_{\mathbb{Y}}\left(\gamma_{i}(s, u)-\ln \left(1+\gamma_{i}(s, u)\right) \lambda(d u)\right)\right) d s=0 .
\end{aligned}
$$

Proof. Consider the function $\ln X_{i}(t)$. By the proof of Theorem 7 , it immediately obtains this result.

To get some further characterizations for the stationary distribution defined by (15), we consider its Laplace transform:

$$
F(\theta):=\int_{0}^{\infty} e^{-\theta y} \pi(d y), \quad \operatorname{Re}(\theta)>0
$$

where $\operatorname{Re}(\theta)$ stands for the real part of complex $\theta$. Recall that $X(t) \in \mathbb{R}_{+}^{n}$ a.s., which implies that the stationary distribution is concentrated on $[0,+\infty)$. By the bounded property of the distribution function, the Laplace transform of stationary distribution is well defined on $\operatorname{Re}(\theta)>0$.

Lemma 9. If $\lim _{t \rightarrow+\infty}(1 / t) \int_{0}^{t} g(s) d s=\bar{g}$ and the process $X(t)$ has a unique stationary distribution $\pi$, then

$$
\lim _{t \rightarrow+\infty} \frac{1}{t} \int_{0}^{t} f(X(s)) g(s) d s=\bar{g} \int_{\mathbb{R}_{+}^{n}} f(x) \pi(d x) \quad \text { a.s. }
$$

Proof. According to the condition $\lim _{t \rightarrow+\infty}(1 / t) \int_{0}^{t} g(s) d s=$ $\bar{g}$, then function $g(s)$ can be rewritten as

$$
\frac{1}{t} \int_{0}^{t} g(s) d s=\bar{g}+\beta(t)
$$

where $\beta(t)$ is infinitesimal; that is, $\lim _{t \rightarrow \infty} \beta(t)=0$. In virtue of equality (33), we have

$$
g(t)=\bar{g}+\beta^{\prime}(t) t+\beta(t)
$$

Consequently,

$$
\begin{aligned}
& \lim _{t \rightarrow+\infty} \frac{1}{t} \int_{0}^{t} f(X(s)) g(s) d s \\
& =\lim _{t \rightarrow+\infty}\left(\frac{\bar{g}}{t} \int_{0}^{t} f(X(s)) d s+\frac{1}{t} \int_{0}^{t} f(X(s)) \beta^{\prime}(s) s d s\right. \\
& \left.\quad+\frac{1}{t} \int_{0}^{t} f(X(s)) \beta(s) d s\right) \\
& :=I_{1}+I_{2}+I_{3} .
\end{aligned}
$$

Note that $\lim _{t \rightarrow \infty} \beta(t)=0$. For any $\varepsilon>0$, there exists a sufficiently large constant $M$, such that for any $t>M$, we have

$$
|\beta(t)|<\varepsilon
$$

Consequently,

$$
I_{3}=\frac{1}{t}\left(\int_{0}^{M} f(X(s)) \beta(s) d s+\int_{M}^{t} f(X(s)) \beta(s) d s\right) \longrightarrow 0
$$

As for the second term, it is not hard to show that $I_{2} \rightarrow 0$. According to the ergodic lemma, we have

$$
I_{1} \longrightarrow \bar{g} \int_{\mathbb{R}_{+}^{n}} f(x) \pi(d x) .
$$

To state our main result, we need the following conditions about the coefficients.

Assumption A6. There exist constants $\bar{a}_{i}, \bar{b}_{i j}, \bar{\sigma}_{i j}, \bar{\gamma}_{i}(u)$ such that

$$
\begin{aligned}
& \lim _{t \rightarrow+\infty} \frac{1}{t} \int_{0}^{t} a_{i}(s) d s=\bar{a}_{i}, \\
& \lim _{t \rightarrow+\infty} \frac{1}{t} \int_{0}^{t} b_{i j}(s) d s=\bar{b}_{i j}, \\
& \lim _{t \rightarrow+\infty} \frac{1}{t} \int_{0}^{t} \sigma_{i j}(s) d s=\bar{\sigma}_{i j}, \\
& \lim _{t \rightarrow+\infty} \frac{1}{t} \int_{0}^{t} \gamma_{i}(s, u) d s=\bar{\gamma}_{i}(u) .
\end{aligned}
$$

We now state the main result of this section.

Theorem 10. Assume that Assumptions A1, A4, A5, and A6 hold. The stationary distribution $\pi$ defined by (15) satisfies, for any complex number $\theta$, with $\operatorname{Re}(\theta)>0$, the following:

$$
\theta \int_{\mathbb{R}_{+}^{n}} e^{-\theta y_{i}} y_{i}\left(\bar{a}_{i}-\sum_{j=1}^{n} \bar{b}_{i j} y_{j}-\int_{\mathbb{Y}} \bar{\gamma}_{i}(u) \lambda(d u)\right) \pi(d y)
$$




$$
\begin{aligned}
= & \frac{1}{2} \theta^{2} \sum_{j=1}^{n} \bar{\sigma}_{i j}^{2} \int_{\mathbb{R}_{+}^{n}} e^{-\theta y_{i}} y_{i}^{2} \pi(d y) \\
& +\int_{\mathbb{R}_{+}^{n}} \int_{\mathbb{Y}}\left(e^{-\theta y_{i}\left(1+\bar{\gamma}_{i}(u)\right)}-e^{-\theta y_{i}}\right) \lambda(d u) \pi(d y) .
\end{aligned}
$$

Proof. Let $f(z)=e^{-\theta z}, 0<\operatorname{Re}(\theta)<+\infty, 0 \leq z<+\infty$. By Itô's formula, we have

$$
\begin{aligned}
d e^{-\theta X_{i}(t)}= & -\theta e^{-\theta X_{i}(t)} d X_{i}^{c}(t) \\
& +\frac{1}{2} \theta^{2} e^{-\theta X_{i}(t)}\left(X_{i}^{c}(t)\right)^{2} \sum_{j=1}^{n} \sigma_{i j}^{2}(t) d t \\
& +\int_{Y}\left(e^{-\theta X_{i}(t)\left(1+\gamma_{i}(t, u)\right)}-e^{-\theta X_{i}(t)}\right) N(d t, d u),
\end{aligned}
$$

where $X_{i}^{c}(t)$ stands for the continuous part of the process $X_{i}(t)$. In integral form, we get

$$
\begin{aligned}
e^{-\theta X_{i}(t)}= & e^{-\theta X_{i}(0)} \\
& -\theta \int_{0}^{t} e^{-\theta X_{i}(s)} X_{i}(s)\left(a_{i}(s)-\sum_{j=1}^{n} b_{i j}(s) X_{j}(s)\right. \\
& \left.-\int_{\mathbb{Y}} \gamma_{i}(s, u) \lambda(d u)\right) d s \\
& +\frac{1}{2} \theta^{2} \int_{0}^{t} e^{-\theta X_{i}(s)}\left(X_{i}(s)\right)^{2} \sum_{j=1}^{n} \sigma_{i j}^{2}(s) d t \\
& +\int_{0}^{t} \int_{\mathbb{Y}}\left(e^{-\theta X_{i}(s)\left(1+\gamma_{i}(s, u)\right)}-e^{-\theta X_{i}(s)}\right) \lambda(d u) d s \\
& -\theta M_{5}(t)+M_{6}(t),
\end{aligned}
$$

where

$$
\begin{gathered}
M_{5}(t)=\int_{0}^{t}\left(e^{-\theta X_{i}(s)} X_{i}(s) \sum_{j=1}^{n} \sigma_{i j}(s)\right) d W_{j}(s), \\
M_{6}(t)=\int_{0}^{t} \int_{\mathbb{Y}}\left(e^{-\theta X_{i}(s)\left(1+\gamma_{i}(s, u)\right)}-e^{-\theta X_{i}(s)}\right) \widetilde{N}(d s, d u) .
\end{gathered}
$$

By Theorem 6 and Assumption A4, it is easy to see the sharp bracket process:

$$
\left\langle M_{5}\right\rangle(t)<K t, \quad\left\langle M_{6}\right\rangle(t)<K t \quad \text { a.s. }
$$

Hence, by the strong law of large numbers of martingales,

$$
\begin{array}{ll}
\lim _{t \rightarrow \infty} \frac{M_{5}(t)}{t}=0, & \text { a.s., } \\
\lim _{t \rightarrow \infty} \frac{M_{6}(t)}{t}=0 & \text { a.s. }
\end{array}
$$

Therefore, by dividing both sides by $t$ of equality (42) and then letting $t \rightarrow \infty$, one gets, by Lemmas 5 and 9 and Theorem 6 , that the assertion (40) holds.

Because Theorem 10 holds for all $\operatorname{Re}(\theta)>0$, one can get a series equality with different values of $\theta$. In particular, If we let $\theta \rightarrow 0$ on the both sides of (40), then we immediately obtain a characterization for covariance matrix of the stationary distribution.

The next result presents the characterization of expectation of the stationary distribution.

Corollary 11. If conditions A1, A5, and A6 hold, then the covariance matrix $\Sigma=\left(\Sigma_{i j}\right)_{n \times n}$ of the stationary distribution obeys

$$
\bar{a}_{i} \bar{\mu}_{i}-\sum_{j=1}^{n} \bar{b}_{i j}\left(\Sigma_{i j}+\bar{\mu}_{i} \bar{\mu}_{j}\right)=0, \quad 1 \leq i \leq n .
$$

Proof. By Theorem 10, the stationary distribution $\pi$ satisfies

$$
\begin{aligned}
\int_{\mathbb{R}_{+}^{n}} e^{-\theta y_{i}} y_{i}\left(\bar{a}_{i}-\sum_{j=1}^{n} \bar{b}_{i j} y_{j}-\int_{\mathbb{Y}} \bar{\gamma}_{i}(u) \lambda(d u)\right) \pi(d y) \\
=\frac{1}{2} \theta \sum_{j=1}^{n} \bar{\sigma}_{i j}^{2} \int_{\mathbb{R}_{+}^{n}} e^{-\theta y_{i}} y_{i}^{2} \pi(d y) \\
+\frac{1}{\theta} \int_{\mathbb{R}_{+}^{n}} \int_{\mathbb{Y}}\left(e^{-\theta y\left(1+\overline{\gamma_{i}}(u)\right)}-e^{-\theta y}\right) \lambda(d u) \pi(d y) .
\end{aligned}
$$

By letting $\theta \downarrow 0$, we get

$$
\begin{gathered}
\int_{\mathbb{R}_{+}^{n}} y_{i}\left(\bar{a}_{i}-\sum_{j=1}^{n} \bar{a}_{i j} y_{j}-\int_{\mathbb{Y}} \bar{\gamma}_{i}(u) \lambda(d u)\right) \pi(d y) \\
=-\int_{\mathbb{R}_{+}^{n}} \int_{\mathbb{Y}} y_{i} \bar{\gamma}_{i}(u) \lambda(d u) \pi(d y) .
\end{gathered}
$$

This completes the proof.

Remark 12. Mao [7] obtains the result (46) for the facultative model without jumps with nonsingular noise coefficients and Tong et al. [5] obtain the result (46) for the facultative model without jumps with singular noise coefficients.

Corollary 13. Under Assumptions A1, A5, and A6, for $k \in \mathbb{N}^{+}$, we have

$$
\begin{aligned}
k \int_{\mathbb{R}_{+}^{n}} y_{i}^{k}\left(\overline{a_{i}}-\sum_{j=1}^{n} \bar{b}_{i j} y_{j}\right) \pi(d y) \\
\quad+\frac{1}{2} k(k-1) \sum_{j=1}^{n} \bar{\sigma}_{i j}^{2} \int_{\mathbb{R}_{+}^{n}} y_{i}^{k} \pi(d y) \\
\quad+\int_{\mathbb{R}_{+}^{n}} \int_{\mathbb{Y}} y_{i}^{k}\left[\left(1+\bar{\gamma}_{i}(u)\right)^{k}-1\right] \lambda(d u) \pi(d y)=0 .
\end{aligned}
$$


Proof. By virtue of Theorem 6, the $k$ th moment of stationary distribution $\int_{\mathbb{R}_{+}^{n}}|x|^{k} \pi(d x)$ is well defined. Expanding the exponential function $\exp (-\theta x)$ into the power series and comparing the terms with the same powers of $\theta^{k}$, one can obtain the equality (49) immediately.

\section{Conflict of Interests}

The authors declare that there is no conflict of interests regarding the publication of this paper.

\section{Acknowledgments}

The authors are grateful to the anonymous referees for their valuable comments and suggestions which led to improvements in this paper. Research of the authors was partially supported by National Natural Science Foundation of China (nos. 11171062, 11101077, 11071258, 11101054, and 11201062), the Fundamental Research Funds for the Central Universities, and the Innovation Program of Shanghai Municipal Education Commission (no. 12ZZ063).

\section{References}

[1] J. Bao, X. Mao, G. Yin, and C. Yuan, "Competitive LotkaVolterra population dynamics with jumps," Nonlinear Analysis: Theory, Methods \& Applications, vol. 74, no. 17, pp. 6601-6616, 2011.

[2] J. Bao and C. Yuan, "Stochastic population dynamics driven by Lévy noise," Journal of Mathematical Analysis and Applications, vol. 391, no. 2, pp. 363-375, 2012.

[3] I. Karatzas and S. E. Shreve, Brownian Motion and Stochastic Calculus, vol. 113 of Graduate Texts in Mathematics, Springer, New York, NY, USA, 2nd edition, 1991.

[4] R. N. Bhattacharya and E. C. Waymire, Stochastic Processes with Applications, Wiley Series in Probability and Mathematical Statistics: Applied Probability and Statistics, John Wiley \& Sons, New York, NY, USA, 1990.

[5] J. Tong, Z. Zhang, and J. Bao, "The stationary distribution of the facultative population model with a degenerate noise," Statistics \& Probability Letters, vol. 83, no. 2, pp. 655-664, 2013.

[6] R. Z. Has'minskiǔ, Stochastic Stability of Differential Equations, vol. 7 of Monographs and Textbooks on Mechanics of Solids and Fluids: Mechanics and Analysis, Sijthoff \& Noordhoff, Alphen aan den Rijn, The Netherlands, 1980.

[7] X. Mao, "Stationary distribution of stochastic population systems," Systems \& Control Letters, vol. 60, no. 6, pp. 398-405, 2011. 


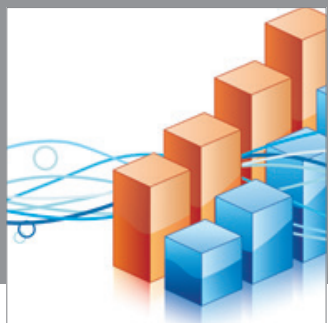

Advances in

Operations Research

mansans

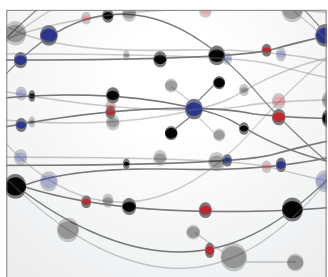

The Scientific World Journal
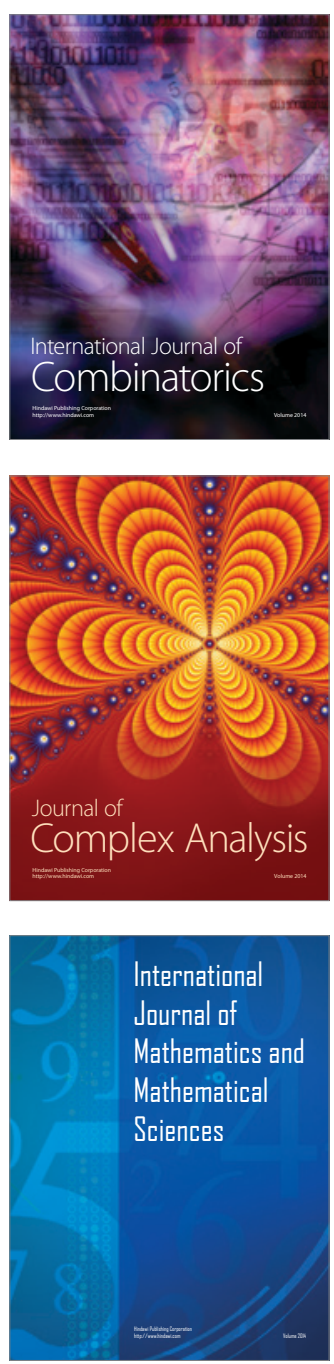
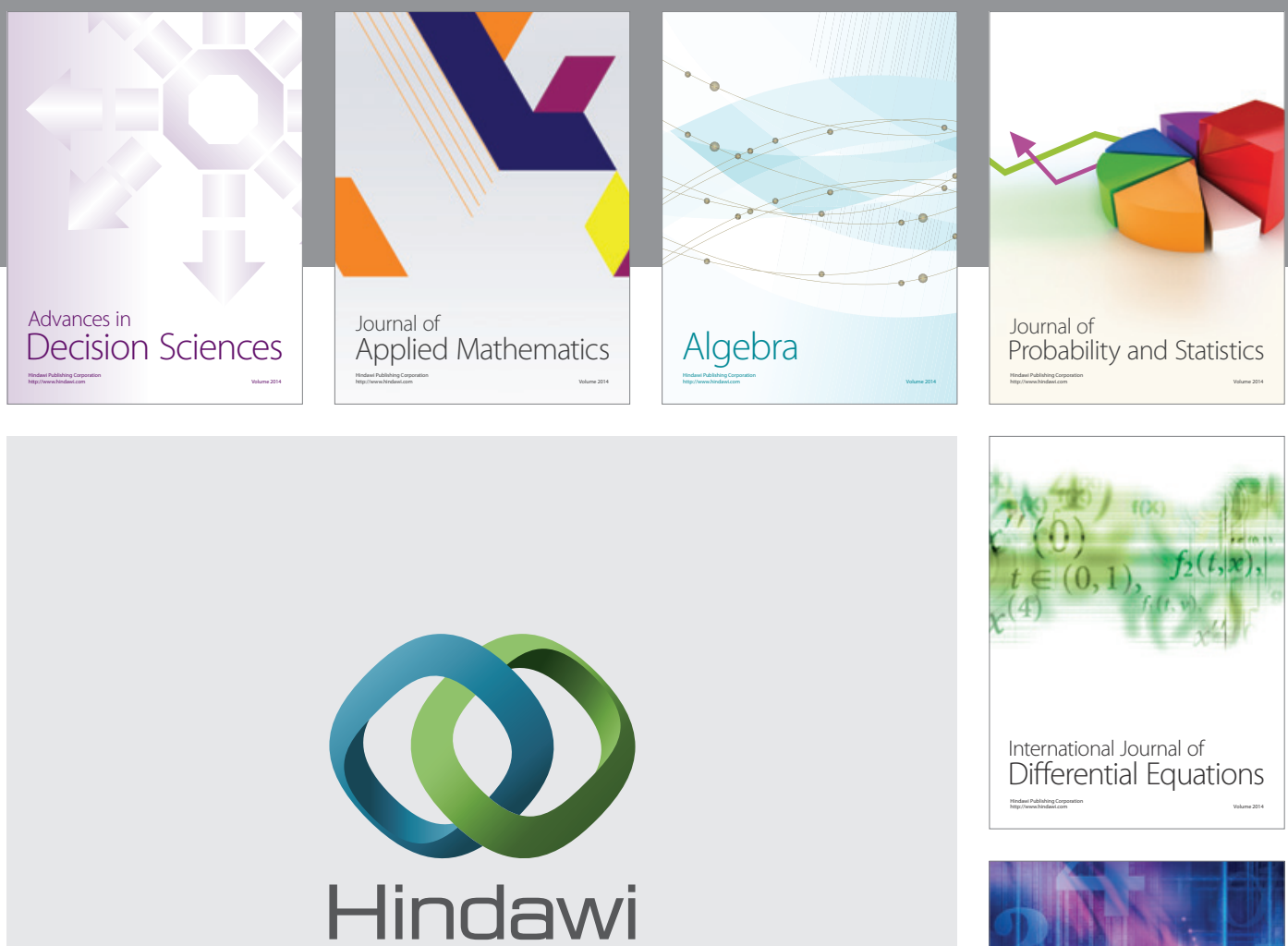

Submit your manuscripts at http://www.hindawi.com
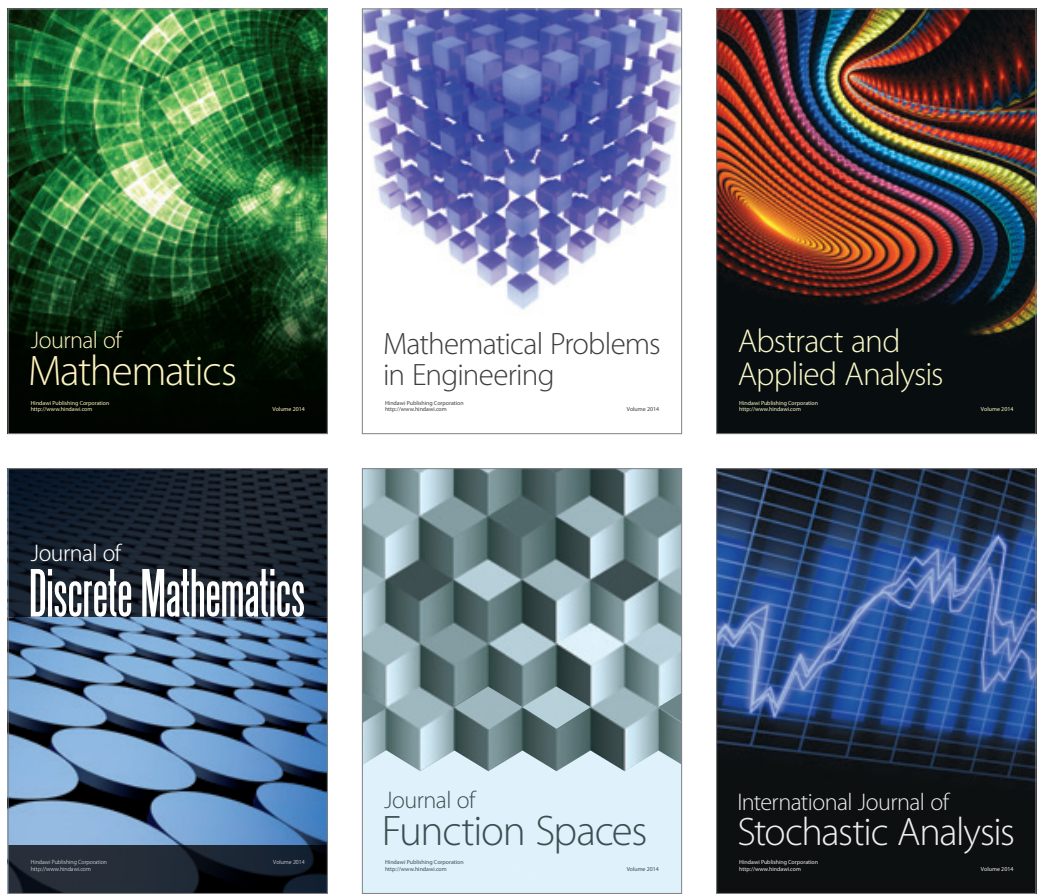

Journal of

Function Spaces

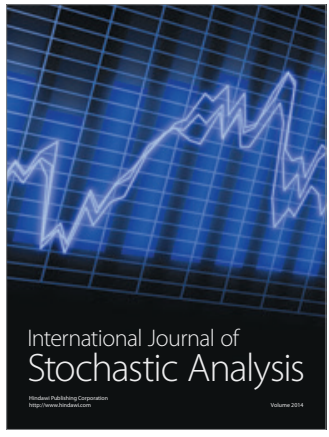

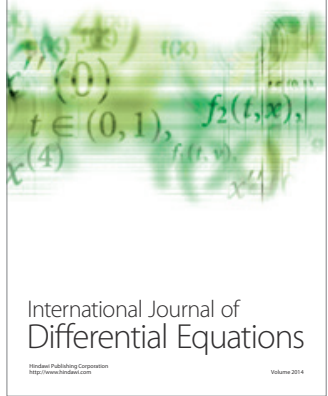
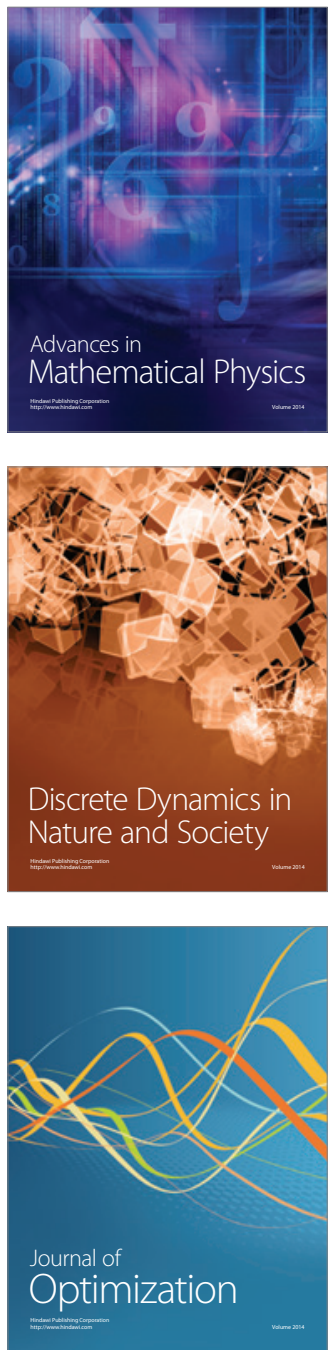